\title{
Enhancement of Luminous Power and Efficiency in InGaN/GaN based Light Emitting Diode using High- $\mathrm{K}$ Dielectric Material
}

saranya G ( $\sim$ saranyagrajan@gmail.com )

Karunya Institute of Technology and Sciences

Sivamangai $\mathbf{N}$ M

Karunya Institute of Technology and Sciences

\section{Research Article}

Keywords: LED, GaN, High-K Dielectric, InGaN, IQE, HfO2

Posted Date: May 10th, 2021

DOl: https://doi.org/10.21203/rs.3.rs-470351/v1

License: (c) (1) This work is licensed under a Creative Commons Attribution 4.0 International License.

Read Full License

Version of Record: A version of this preprint was published at Optical and Quantum Electronics on July 22nd, 2021. See the published version at https://doi.org/10.1007/s11082-021-03054-z. 


\title{
Enhancement of Luminous Power and Efficiency in InGaN/GaN-LED using High-K Dielectric Material
}

\author{
Saranya G and Sivamangai N M \\ Karunya Institute of Technology and Sciences, Coimbatore and India
}

\begin{abstract}
A novel high-k dielectric material is proposed for InGaN/GaN-LED to improve the performance. The proposed LED is analyzed and benchmarked with conventional LED using Technological Computer Aided Design (TCAD). The qualitative consistency of the optical characteristics of LED in this work has been observed that validate the TCAD simulation. Further, it is observed that proposed LED recorded higher luminous power and internal quantum efficiency (IQE) than that of conventional LED. At the injection current of $600 \mathrm{~mA}$, the proposed and conventional LED yielded luminous power of $1600 \mathrm{~mW}$ and $1400 \mathrm{~mW}$, respectively. Further, IQE of the proposed LED is higher than that of conventional LED by $12 \%$. The improvement in the optical performance is attribute to high-k dielectric material induced additional electric field and radiative recombination rate. Thus, the proposed LED with high-k dielectric material is an outstanding device in lightning application.
\end{abstract}

Keywords-LED, GaN, High-K Dielectric, InGaN, IQE, $\mathrm{HfO}_{2}$

\begin{tabular}{cl} 
& \multicolumn{1}{c}{ NoMENCLATURE } \\
$n_{i}$ & Intrinsic carrier concentration \\
$p$ & Hole concentration \\
$n$ & Electron concentration \\
$q$ & Electronic charge \\
$J_{n}$ & Electron current density \\
$J_{h}$ & Hole current density \\
$\mu_{p}$ & Hole mobility \\
$\mu_{n}$ & Electron mobility \\
$\psi$ & Quasi fermi potential \\
$\varepsilon$ & Permittivity \\
$\varphi$ & Electrostatic potential \\
$R_{R A D}$ & Radiative recombination rate \\
$R_{A U G E R}$ & Auger recombination rate \\
$R_{S R H}$ & Shockley-Read-Hall recombination rate \\
$\tau_{n}$ & Electron life-time \\
$\tau_{p}$ & Hole life-time \\
$F_{C}$ & Electric field \\
$K$ & Boltzmann constant \\
$T$ & Lattice temperature
\end{tabular}

\section{INTRODUCTION}

In the past few decade, the research in GaN based LED has demonstrated impressive advancements. It is due to the excellent material property. Further, these properties facilitate $\mathrm{GaN}$ based LED to perform well in color display, automobile/home interior-lightning and running light. The performance of the LED in these application depends on active layer, body thickness and crystal plane [1-5]. The active layer consists of barrier and potential well. LED with multiple well tend to have higher Internal Quantum Efficiency (IQE) and luminous power [6-8]. The well thickness lower than 10 $\mathrm{nm}$ allow electrons to exist at discrete energy levels and better confinement. This leads to the enhancement of quantum efficiency. However, increasing number of quantum well more than 10 is not desirable since additional fabrication steps and its growth related defects. On the other hand, ultra-thin body based LED received impressive attention in inter/intrachip interconnects [3-5]. Further, the M-plane also enhances the IQE compared to c-plane [2]. In addition to aforementioned advancement, Electron Blocking Layer (EBL) also improve the efficiency as a result of reduced carrier over-flow. However, a lot of research have been carried out on barrier design to improve the LED performance [9-14]. Furthermore, analyzing and improving efficiency droop in light emitting diode also received a great attention in the last few decades [15-19]. Although, GaN-LED has shown improvements in efficiency and output power, it is still suffering from higher temperature, carrier-leakage, Auger recombination, micro-cracks and poor hole injection. The elevated temperature due to recombination process has aging effect and reduces the device reliability for long life-time application. In addition, the higher temperature and material intrinsic defects induces micro-cracks which is physical damage [20-21]. The defects or micro-cracks is originating from lattice mismatch at nitride/substrate interface. Silicon (Si) and Sapphire substrate induce more dislocation and defects at Nitride/substrate interface. SiC emerged as an alternative substrate to $\mathrm{Si}$ and Sap as the manifestation of lower lattice mismatch and its corresponding lower microcracks or defects at Nitride/SiC interface. Thus, it is important to selected substrate and mitigate crack/defects as it is a serious concern in recombination process and efficiency droop. Micro-cracks enhance SRH non-radiative recombination through trap centers. The larger non-radiative recombination results in lower radiative recombination which is not desirable. Further, non-radiative recombination generates more phonons rather than photon and thereby increases the device self-heating and degrades the device reliability. Thus, the performance of the LED degraded as the manifestation of reduced radiative recombination and device self-heating. Hence, it is prime importance to enhance luminous power and quantum efficiency in LED. In this paper, novel high-k dielectric material technique is introduced in the GaN LED to enhance the performance. Impact of dielectric material on the performance is analyzed using Technology Computer Aided Design (TCAD) simulation. Optical characteristics trend shows a qualitative consistency with 
reported works that ensure the validation of the simulation. Impact of dielectric material on luminous power, electric field and quantum efficiency is also analyzed.

\section{DEVICE DESCRIPTION}

The conventional and proposed GaN LED is shown in Fig. 1 (a) and Fig. 1 (b), respectively. The GaN LED consists of 500-nm-thick GaN, 100-nm-thick AlGaN electron blocking layer, four 3-nm-thick InGaN well, three 5-nm-thick GaN well, 3.375- $\mu$ m-thick GaN Buffer. Sapphire material is used as a substrate with thickness of 6 micrometre. Compared to conventional LED, the proposed LED has additional $\mathrm{HfO}_{2}$ layer to enhance optical performance.

The role of $\mathrm{HfO} 2$ is to modify the electric field around quantum well such that carrier concentration and recombination are enhanced in the well. The $\mathrm{HfO}_{2}$ layer is defined in the simulation using region statement in ATLAS TCAD deckbuild. Band-gap and dielectric constant of $\mathrm{HfO}_{2}$ are $6 \mathrm{eV}$ and 22, respectively. The $\mathrm{HfO}_{2}$ length is $25 \mu \mathrm{m}$ and thickness is $1.5 \mu \mathrm{m}$. The field modulation and enhancement of recombination could be done by $\mathrm{HfO}_{2}$ material. Further, in fabrication, $\mathrm{HfO} 2$ needs deposition method rather than growth method. Deposition is simple process compared to the growth process. The difference between low $\mathrm{K}$ and high $\mathrm{K}$ material is dielectric constant. The variation in the dielectric constant results in variation of electric field across it. Therefore, the low $\mathrm{K}$ and high $\mathrm{K}$ material tend to have variation in electric field. The electron blocking layer is used to reduce the electron leakage. For $n-G a N$ and $p-G a N$, the $n$ and $\mathrm{p}$ doping are $1 \times 10^{18} \mathrm{~cm}^{-3}$ and $1 \times 10^{19} \mathrm{~cm}^{-3}$, respectively. The mole fraction (Aluminium composition) used in the EBL layer is $20 \%$. Multiple quantum well is used to enhance the recombination. Thick GaN buffer is used to reduce defect propagation from substrate/buffer interface.

\section{Simulation PHYSICS}

Simulation of the conventional and proposed device are carried out using ATLAS TCAD. Various physical models

\section{Conventional LED}

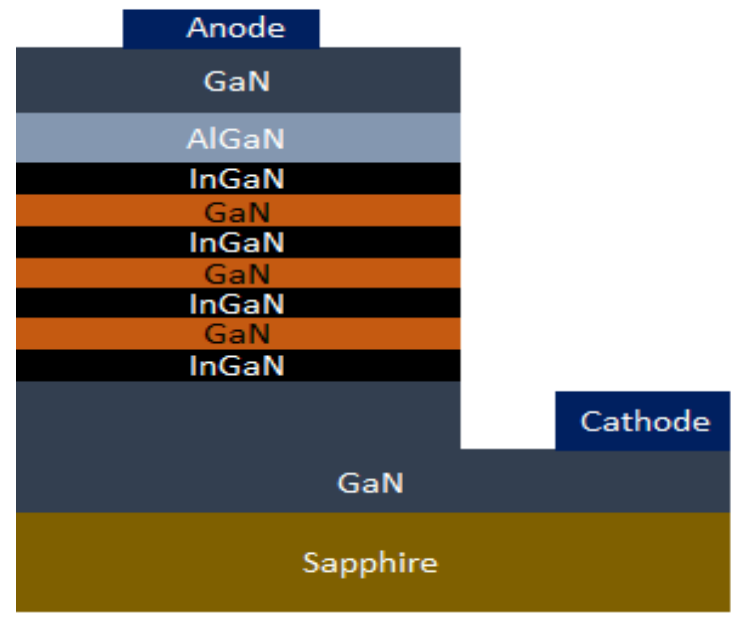

are used to account recombination process and polarization effect in the simulation. The carrier transport in the LED is governed using Drift-Diffusion (DD) model in the simulation. In the DD model, the electron $\left(J_{h}\right)$ hole $\left(J_{h}\right)$ current density are expressed as

$J_{h}=p q \mu_{p} \nabla \theta$

$J_{n}=n q \mu_{n} \nabla \theta$

The description of all model parameters used in the simulation is given in above nomenclature section.

On the other hand, the Poison model is facilitated in the simulation to account the effect of carrier distribution over the space, which is denoted as

$\nabla(\varepsilon \nabla \varphi)=-\rho$

However, the Poison model is not enough since the GaN LED is more relevant to quantum effect. Thus, the Schrodinger model is augmented with Poison model to account the electron/hole quantum behavior. Apart from fundamental models, Shockley-Read-Hall (SRH), Auger, and radiative models are used to govern carrier generation and recombination process in potential quantum well. The radiative $\left(R_{R A D}\right)$, auger $\left(R_{A U G E R}\right)$ and SRH $\left(R_{S R H}\right)$ models are expressed as

$$
\begin{aligned}
& R_{R A D}=A\left(n p-n_{i}{ }^{2}\right) \\
& R_{A U G E R}=\left(A_{n} n+A_{p} p\right)\left(n p-n_{i}{ }^{2}\right) \\
& R_{S R H}=\frac{n p-n_{i}^{2}}{\tau_{n}\left(p+n_{i} \exp \left[\frac{-F_{C}}{K T}\right]\right)+\tau_{p}\left(n+n_{i} \exp \left[\frac{F_{C}}{K T}\right]\right)}
\end{aligned}
$$

Polarization models are used in the simulation to account the piezoelectric and spontaneous polarization effect. Further, the carrier statistics also included using Fermi-Dirac model.

\section{Proposed LED}

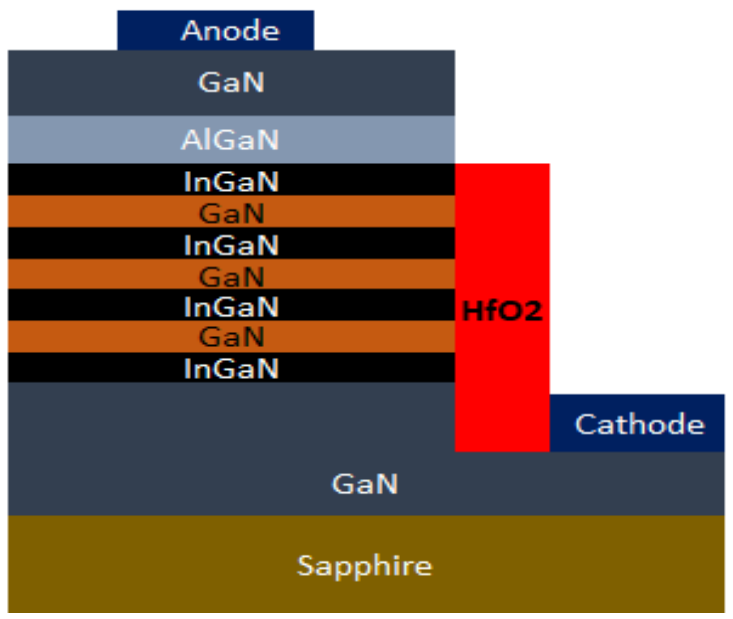

Fig. 1 Conventional and proposed GaN LED. 


\section{RESUlT AND DISSCUSSION}

On investigating the LED using physical TCAD simulator, first, it is mandatory to validate the simulation. Therefore, simulation is carried out to extract the current densities across the active layers of the device. The extracted current densities across the four quantum-well is shown in Fig. 2. Even, the extraction is done in vector component of the current densities rather than its magnitude. It is due to that vectors quantities expose a lot of and interesting intangible physics in the device. As it can be seen in Fig. 2, the following interesting facts has been observed such as: (i) current densities at barrier-well interface is higher, (ii) the current distribution is not uniform over the entire well-thickness and (iii) the current densities gradually increase from $4^{\text {th }}$ well down to $1^{\text {st }}$ well. The gradual increase in the current densities with respect to well numbers is consistent with the reported data in [22]. Thus, from this consistency, it could be stated that the simulation is valid one.

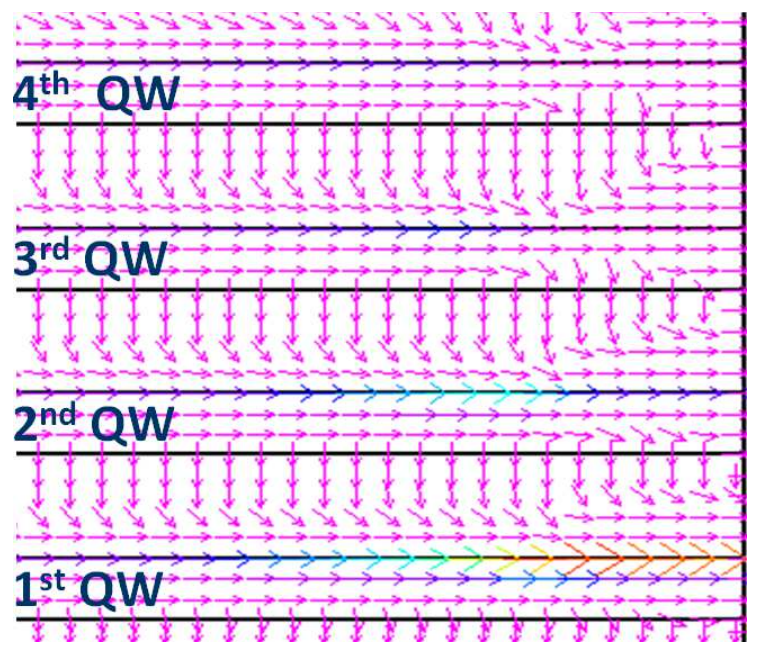

Fig. 2 Current densities at four potential quantum well (QW).

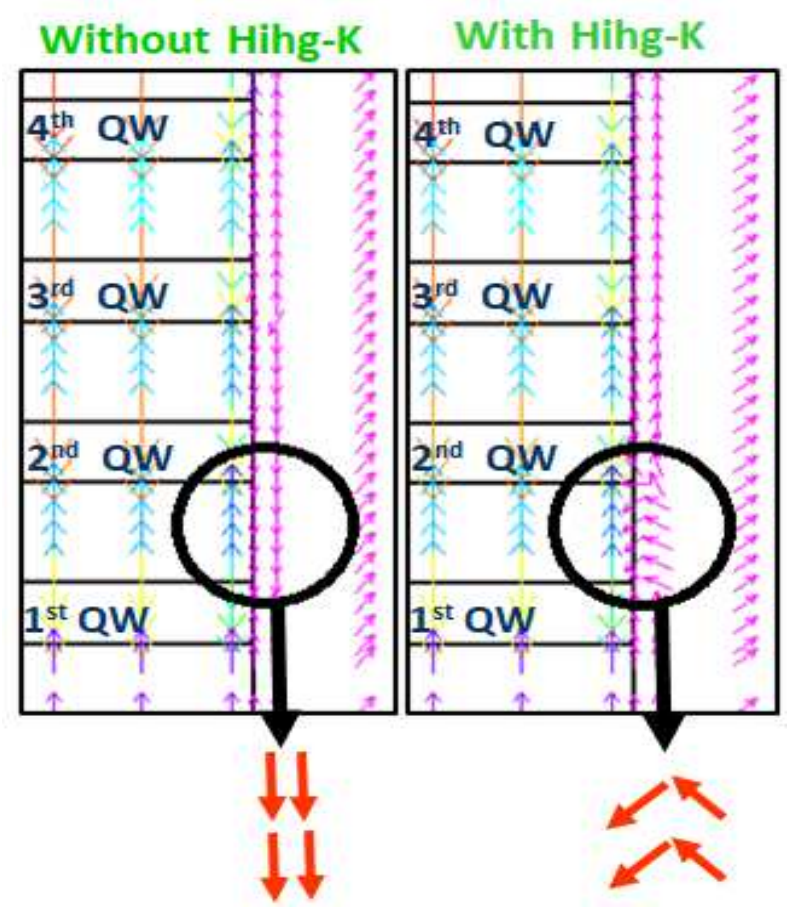

Fig. 3 Electric field distribution of the proposed device and conventional device.
Electric field of the LED is analyzed as it is significantly relative to proposed high-k dielectric technique in the LED. Fig. 3 contrasts the electric field vector of the conventional and proposed LED. It is observed that the electric field converge towards the InGaN/GaN interface and diverge at $\mathrm{GaN} / \mathrm{InGaN}$ interface. It is due to the polarized negative charge at InGaN/GaN interface and positive charge at $\mathrm{GaN} / \mathrm{GaN}$ interface. Therefore, the performed simulation obeys the Gauss law. Furthermore, in contrast to conventional LED, an interesting field modulation is observed in proposed device which is high-lighted using a circle in Fig. 3. In between $1^{\text {st }}$ and $2^{\text {nd }}$ quantum-well of the conventional device, the electric field is in vertical direction. On the other hand, the field in the encircled region of the proposed LED from vertical direction to horizontal direction. It is due to the incorporation of high-k material in the proposed LED. This electric field deflection is important since it has great influence on generation and recombination of the carriers.

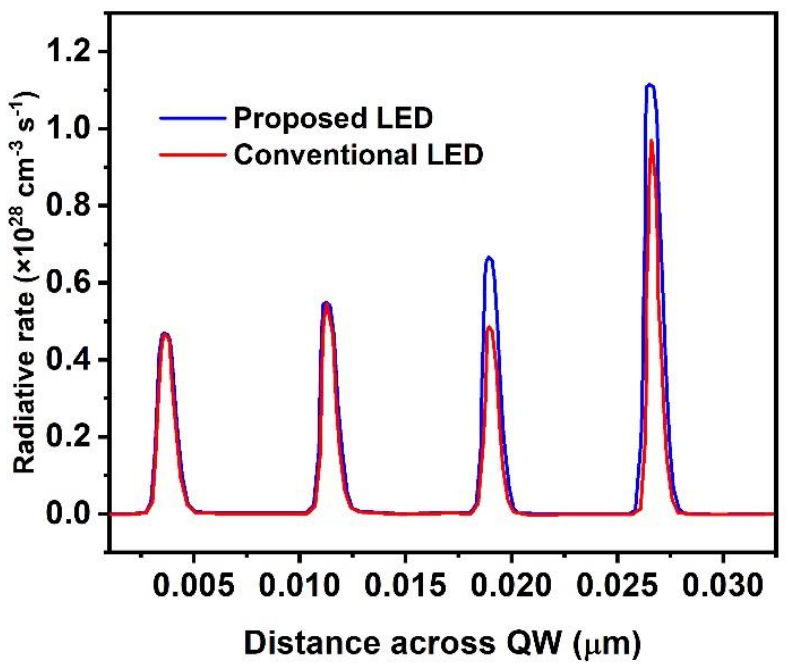

Fig. 4 Radiative recombination rate across quantum well for conventional and proposed LED.

The radiative recombination rate across quantum well of proposed and conventional LED is contrasted in Fig. 4. It is observed that recombination rate is higher in well-region. It is due to higher electron/hole concentration in the quantum-well. In other words, recombination rate in the quantum well is proportional to the product of hole and electron concentration. Further, it is observed that the recombination is higher towards the $\mathrm{P}-\mathrm{GaN}$ and it is due to higher hole injection in the quantum well which is closer to $\mathrm{P}-\mathrm{GaN}$ layer. In addition, it is interesting to note that the recombination rate is higher in two well of proposed LED than that of conventional LED. It is a welcome feature and desirable in the proposed LED as it enhances the quantum efficiency and luminous power. 


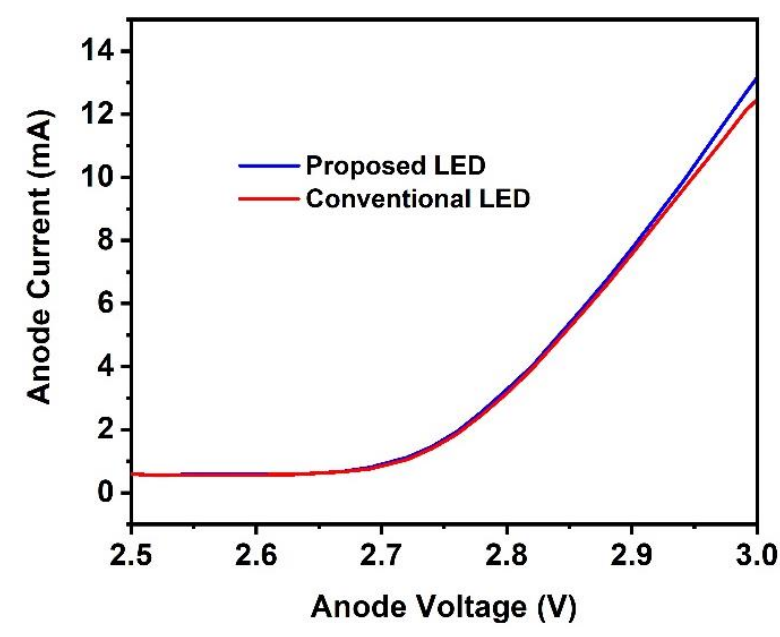

Fig. 5 Anode current at forward bias condition of the LED

The current-voltage (I-V) characteristic a basis for any diode based device to describe its behaviors. Thus, the anode current is observed over Anode voltage, which is shown in Fig. 5. The anode voltage is swept in forward bias region and anode current is observed. The observation shows that the I-V characteristics trend of devices in this paper shows a qualitative consistency with I-V data reported in [23]. From Fig. 5, it has been seen that current of proposed device is same that of conventional device for all bias regime. However, a small discrepancy in current at the anode voltage of 3.0V. The discrepancy could be used to relate the intrinsic series resistance of the device. In contrast to conventional device, a slight improvement in current for proposed device at $3 \mathrm{~V}$ results from lower series resistance.

The luminous power over injection current for proposed device and conventional device is depicted in Fig. 6. For both device, the same size of $1 \mathrm{~mm} \times 1 \mathrm{~mm}$ is considered in the simulation to provide quantitative comparison. Proposed and conventional LED exhibited $1600 \mathrm{~mW}$ and $1400 \mathrm{~mW}$ at the injection current of $600 \mathrm{~mA}$, respectively. It is interesting to note that the luminous power of proposed device is higher than conventional device by $200 \mathrm{~mW}$. This substantial improvement in the light output power for the proposed device is attributed to dielectric material induced recombination.

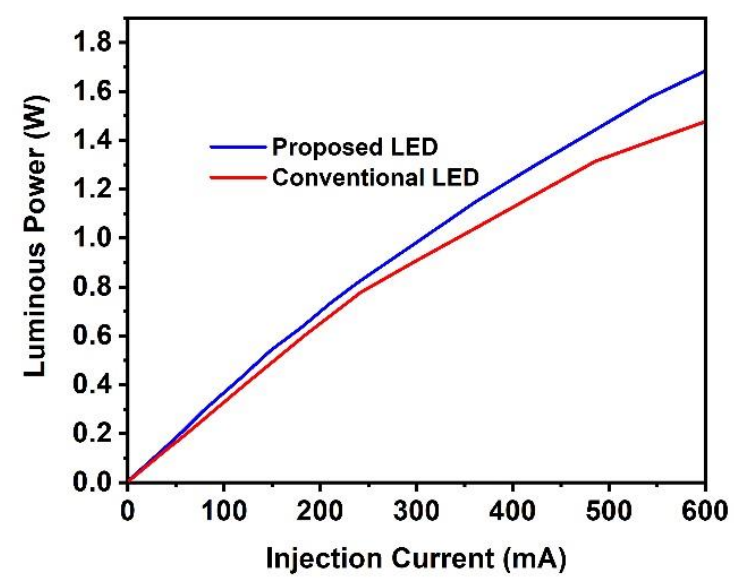

Fig. 6 Luminous Power versus injection current with chip size of $1 \mathrm{~mm} \times 1 \mathrm{~mm}$.

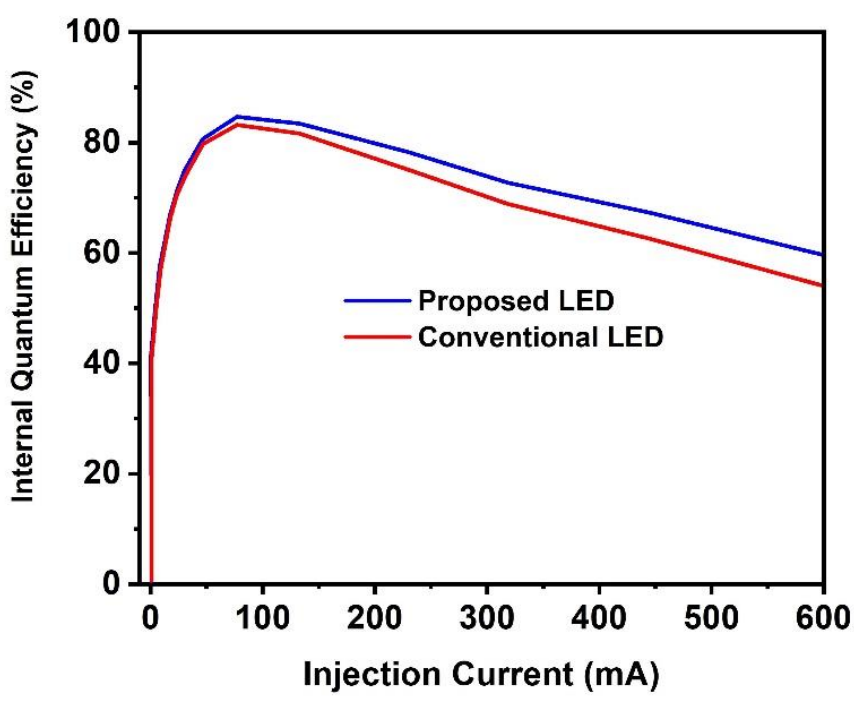

Fig. 7 Internal quantum efficiency over injection current of the InGaN/GaN LED.

Internal Quantum Efficiency is one of the important figure of merit to know-how of the device fit for specific application. Thus, the IQE is extracted for both conventional and proposed LED. It is extracted using the method that radiative recombination rate divided by all recombination rate, which is expressed as

$$
I Q E=\frac{B n^{2}}{A n+B n^{2}+C n^{3}}
$$

where, $\mathrm{A}, \mathrm{B}$ and $\mathrm{C}$ are $\mathrm{SRH}$, radiative and Auger recombination coefficients, respectively. The extracted IQE against injection current is shown in Fig. 7. At lower injection current, both device exhibits the same efficiency. And it peaks up at the injection current of $100 \mathrm{~mA}$. Beyond $100 \mathrm{~mA}$, a droop in internal quantum efficiency is observed. The Augur recombination process at higher injection current is responsible for droop or degradation in efficiency. Although the droop occurs, the IQE of the both device is still comparable. At the higher injection current of $600 \mathrm{~mA}$, the IQE of the proposed LED is higher than that of conventional device by $12 \%$. The observed substantial improvement in IQE for the proposed device is due to high-k dielectric material induced higher electric field and recombination process around the quantum well. The improved IQE enable the device to yield higher luminous power with reduced power consumption.

In a fabricated real device, physical parameters such as well-thickness and well-depth are not necessary as expected all time. Hence, there might be inhomogeneity in the physical parameters and composition too. The Photoluminous (PL) intensity over a range of wavelength reflects the wellthickness, composition and well-depth. Further, the energy band profile and trap activation energy could be extracted from PL spectra. The device technologist in industry also finetune the fabrication process from PL spectra to obtain highfidelity quantum-wells. Thus, analyzing PL spectra is of prime importance. Fig. 8 shows the PL intensity versus wavelength. It is found that both proposed and conventional device peaks at $0.45 \mu \mathrm{m}$ or $450 \mathrm{~nm}$. The peak at $450 \mathrm{~nm}$ implies that both device has blue emission. Additionally, it is 
found that the peak value of proposed device is higher than the conventional device. It is due to higher injection current in the device.

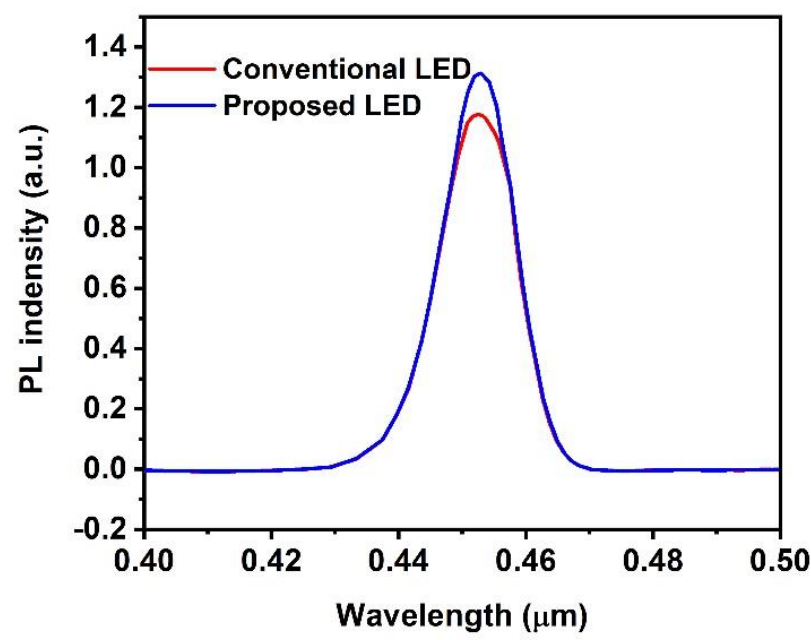

Fig. 8 Photo-luminous (PL) intensity versus wave length of the LED.

In order to provide a better clarity on the performance of the LEDs, the proposed LED is contrasted with conventional LED in table I. The luminous power and IQE in the table is extracted at the injection current of $600 \mathrm{~mA}$. It is clear that the luminous power and IQE of the proposed LED is superior than that of conventional LED.

TABLE I PERFORMANCE METRICS OF THE LED AT $600 \mathrm{~mA}$

\begin{tabular}{l|cc}
\hline Device & Luminous Power (mW) & IQE (\%) \\
\hline Convectional LED & 1400 & 53 \\
Proposed LED & 1600 & 60 \\
\hline
\end{tabular}

\section{CONCLUSSION}

GaN-LED with a novel high-k dielectric technique is proposed and investigated using TCAD physical simulator. Radiative/non-radiative recombination, mobility and carrier statistics models are used to carry out the simulation. The high-k dielectric technique in proposed LED has the advantage of electric field modulation and enhanced recombination rate in the quantum-well compared to conventional LED. Further, the luminous power, IQE, PL intensity over wavelength, current densities and radiative recombination rate are extracted and a qualitatively consistency with reported data has been observed. In contrast to conventional LED, enhancement of luminous power and IQE in proposed LED is achieved.

\section{ACKNOWLEDGMENT}

This work is supported by Karunya Institute of Technology and Sciences, Coimbatore, India.

\section{REFERENCES}

[1] Matteo Meneghini, Nicola Trivellin, Gaudenzio Meneghesso, Enrico Zanoni, Ulrich Zehnder, and Berthold Hahn, "A combined electrooptical method for the determination of the recombination parameters in InGaN-based light-emitting diodes," Journal of Applied Physics vol.106, 114508, 2009.

[2] Md Rezaul Karim Nishat, Mayada M. Taher, Shaikh S. Ahmed "Million-atomtight-binding modeling of non-polara-plane InGaN light emitter," Journal of Computational Electronics, vol.17, pp.1630-1639, Jul 2018

[3] Gaurav Gupta, Florian Mema, Raymond J.E. Hueting, Electron-hole bilayer light-emitting device: Concept and operation, Solid State Electronics, vol.168, pp.107726, Jun 2020

[4] Roelkens G, Liu L, Liang D, Jones R, Fang A, Koch B, Bowers J. III$\mathrm{V} /$ silicon photonics for on-chip and intra-chip optical interconnects. Laser Photon Rev, vol. 4, no. 6, pp. 751-79, 2010

[5] Thomson D, Zilkie A, Bowers JE, Komljenovic T, Reed GT, Vivien L, Marris-Morini D, Cassan E, Virot L, Fédéli J-M, et al. Roadmap on silicon photonics. J, vol. 18, pp.7, pp.073003, Opt 2016

[6] Jun Zhang, Xiang-Jing Zhuo, Dan-Wei Li, Lei Yu, Kai Li, YuanWen Zhang, Jia-Sheng Diao, Xing-Fu Wang, and Shu-Ti Li "Effect of Mg Doping in GaN Interlayer on the Performance of Green LightEmitting Diodes," IEEE Photon. Technol. Lett., vol. 27, no. 2, pp. 117120, Jan. 2015 .

[7] Chi-Kang Li, Maarten Rosmeulen, Eddy Simoen, and Yuh-Renn Wu., "Study on the Optimization for Current Spreading Effect of Lateral GaN/InGaN LEDs," IEEE Trans. Electron Devices, Vol. 61, no. 2, pp. 511-517, Feb. 2014

[8] Moon-Hwan Chang, Diganta Das, P.V. Varde and Michael Pecht "Light emitting diodes reliability review," Microelectron. Reliab., vol. 52, pp. 762782, 2012

[9] Yue Shen, Yuanwen Zhang, Lei Yu, Kai Li, Hui Pi, Jiasheng Diao, Wenxiao Hu,Weidong Song, Chongzhen Zhang, Shuti Li, "The advantages of algan-based ultraviolet light-emitting diodes with Al content graded AlGaN barriers", J. Disp. Technol., vol.11, no.9, pp.677-681, 2015.

[10] L.-W. Cheng, C.-Y. Xu, Y. Sheng, C.-S. Xia, W.-D. Hu, W. Lu, "Study on GaN-based light emitting diode with InGaN/GaN/InGaN multilayer barrier", Opt. Quantum Electron., Vol.44, no.35, pp.75-81 (2012).

[11] L. Cheng, S. Wu, Performance enhancement of blue InGaN light emitting diodes with a GaNeAlGaNeGaN last barrier and without an AlGaN electron blocking layer, IEEE J. Quantum Electron, vol.50, no.4, pp.261-266, 2014

[12] Yujue Yang, Lian Zhang, TongboWei, Yiping Zeng, "Advantages of InGaN light emitting diodes with alternating quantum barriers", $J$. Disp. Technol., vol. 11, no.5, pp.456-460, 2015.

[13] Jih-Yuan Chang, Miao-Chan Tsai, Yen-Kuang Kuo, "Advantages of blue InGaN light-emitting diodes with AlGaN barriers", Opt. Lett., vol.35, no.9, pp.1368-70, 2010.

[14] Chang Sheng Xia, Z.M. Simon Li, Wei Lu, Zhi Hua Zhang, Yang Sheng, Li Wen Cheng, "Droop improvement in blue InGaN/GaN multiple quantum well light-emitting diodes with indium graded last barrier", Appl. Phys. Lett., vol.99, no.5, pp. 233501, 2011.

[15] Li-Hong Zhu, Wei Liu, Fan-Ming Zeng, Yu-Lin Gao, Bao-Lin Liu, YiJun Lu, Zhong Chen, "Efficiency droop improvement in InGaN/GaN light-emitting diodes by graded-composition multiple quantum wells", IEEE Photonics J., vol.5, no.2, pp. 8200208-8200208, Apr 2013.

[16] Yuanping Sun, Hongying Guo, Lihua Jin, Yong-Hoon Cho, E.-K. Suh, H.J. Lee, R.J. Choi, Y.B. Hahn, "Optical excitation study on the efficiency droop behaviours of InGaN/GaN multiple-quantum-well structures", Appl. Phys., vol.114, no.4, pp.551-555 2014.

[17] Jinliang Xu, Tianhu Wang, "Efficiency droop improvement for InGaNbased light- mitting diodes with gradually increased In-composition across the active region", Phys. E, vol.52, no, pp.8-13, 2013.

[18] E. Jaehee Cho, Fred Schubert, Jong Kyu Kim, "Efficiency droop in light-emitting diodes: challenges and countermeasures", laser photonics, Rev., vol.7, no.3, pp.408-421, 2013.

[19] Martin F. Schubert, Sameer Chhajed, Jong Kyu Kim, E. Fred Schubert, Daniel D. Koleske, Mary H. Crawford, Stephen R. Lee, Arthur J. Fischer, Gerald Thaler, Michael A. Banas, "Effect of dislocation 
density on efficiency droop in GaInN/GaN light-emitting diodes", Appl. Phys. Lett., vol.91, pp. 231114, 2007.

[20] Egawa, Takashi, Zhang, B., Nishikawa, N., Ishikawa, H., Jimbo, Takashi and Umeno, Masayoshi "InGaN multiple-quantum-well green lightemitting diodes on $\mathrm{Si}$ grown by metalorganic chemical vapor deposition,” J. Appl. Phys., vol. 91, no. 1, pp. 528-530, 2002.

[21] T. Pinnington, D.D. Koleske, J.M. Zahler, C. Ladous, Y.-B. Park, M.H. Crawford, M. Banas, G. Thaler, M.J. Russell, S.M. Olson and Harry A. Atwater "InGaN/GaN multi-quantum well and LED growth on wafer bonded sapphire-on-polycrystalline AlN Substrates by Metal Organic Chemical Vapor Deposition," J. Crystal Growth, vol. 310, pp $25142519,2008$.

[22] Marco Calciati, Michele Goano, Francesco Bertazzi, Marco Vallone, Xiangyu Zhou, Giovanni Ghione, Matteo Meneghini, Gaudenzio Meneghesso, Enrico Zanoni, Enrico Bellotti, Giovanni Verzellesi, Dandan Zhu, and Colin Humphreys, Correlating electroluminescence characterization and physics-based models of InGaN/GaN LEDs: Pitfalls and open issues, Aip Advances, vol.4, pp. 067118, 2014

[23] Silvino José Antuña Presa "Characterization of GaN-based lightemitting diodes," Thesis, Aug 2016 
Figures

Conventional LED

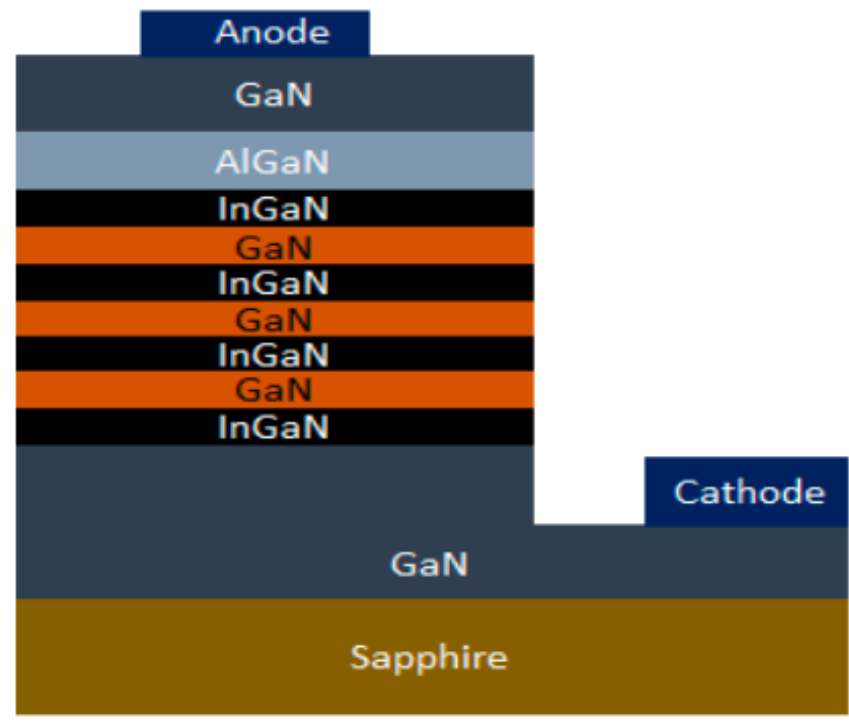

\section{Proposed LED}

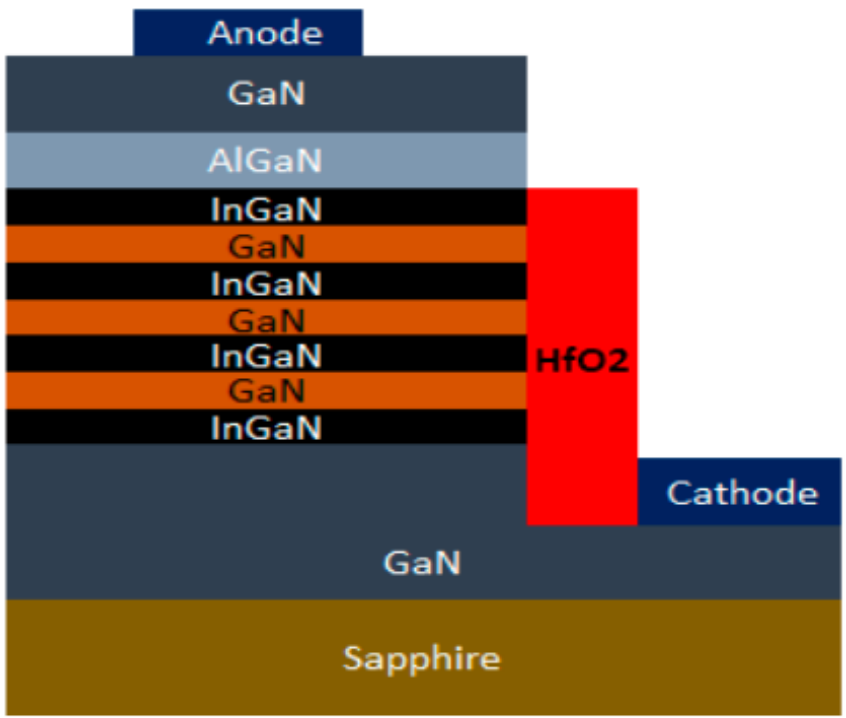

Figure 1

Conventional and proposed GaN LED.

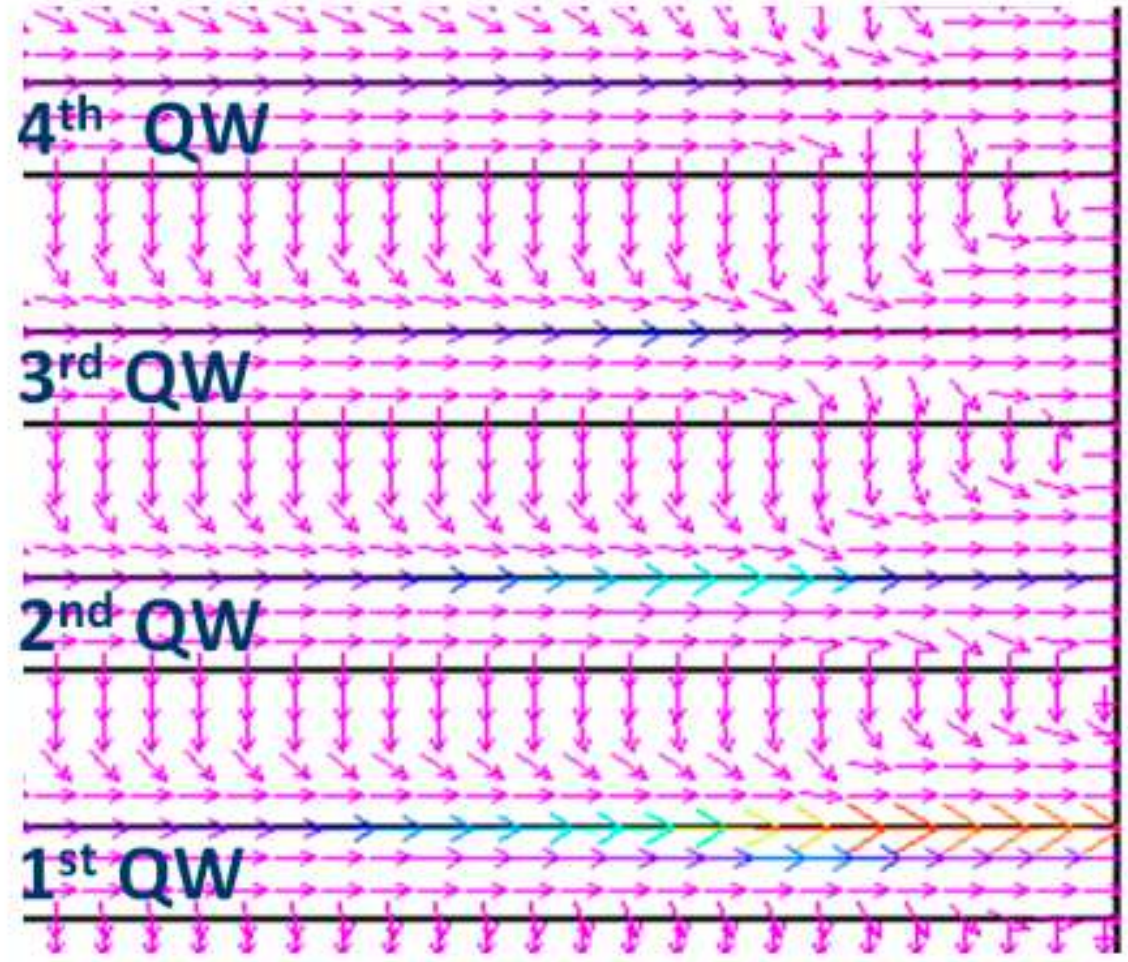

Figure 2

Current densities at four potential quantum well (QW). 


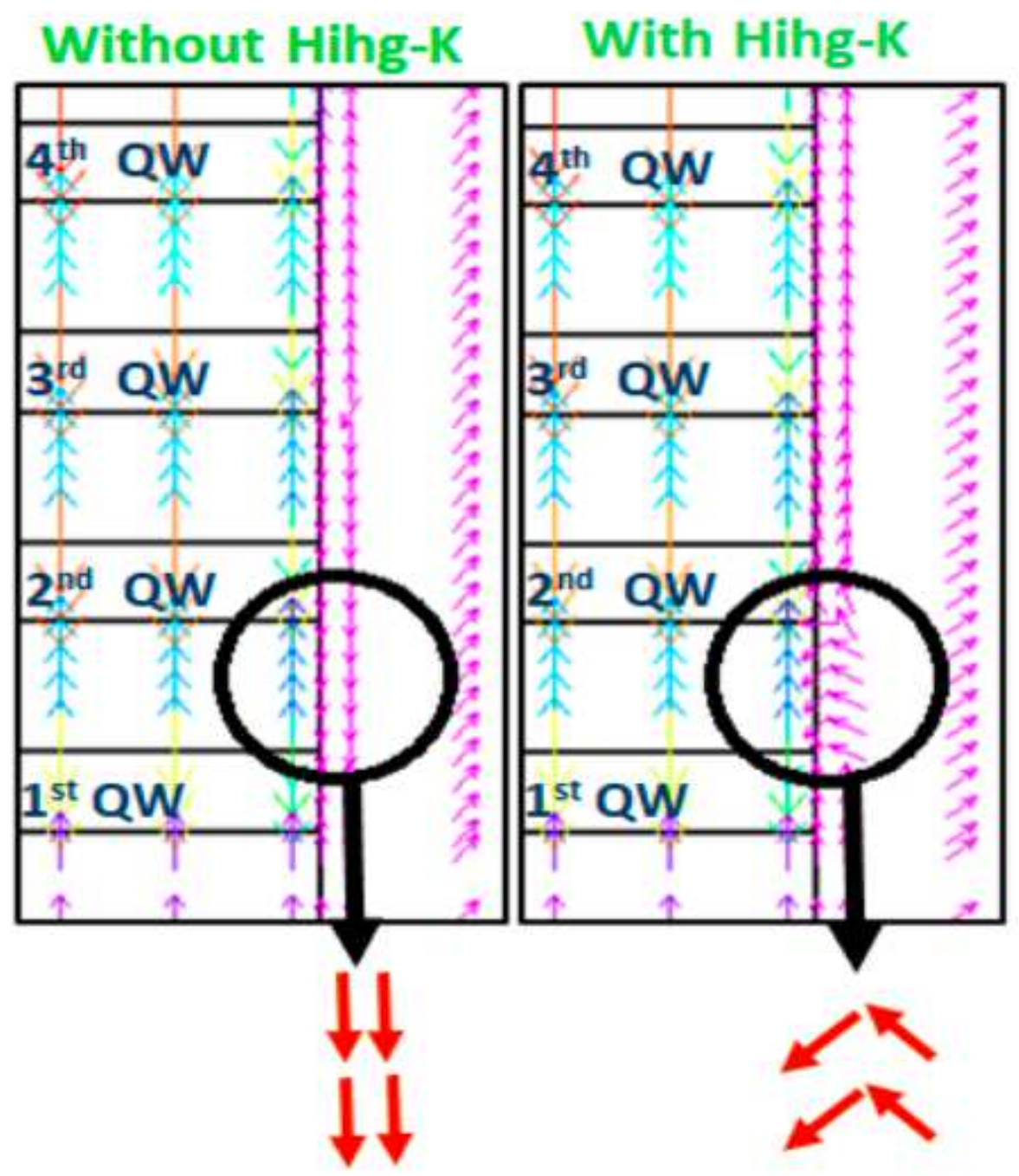

Figure 3

Electric field distribution of the proposed device and conventional device. 


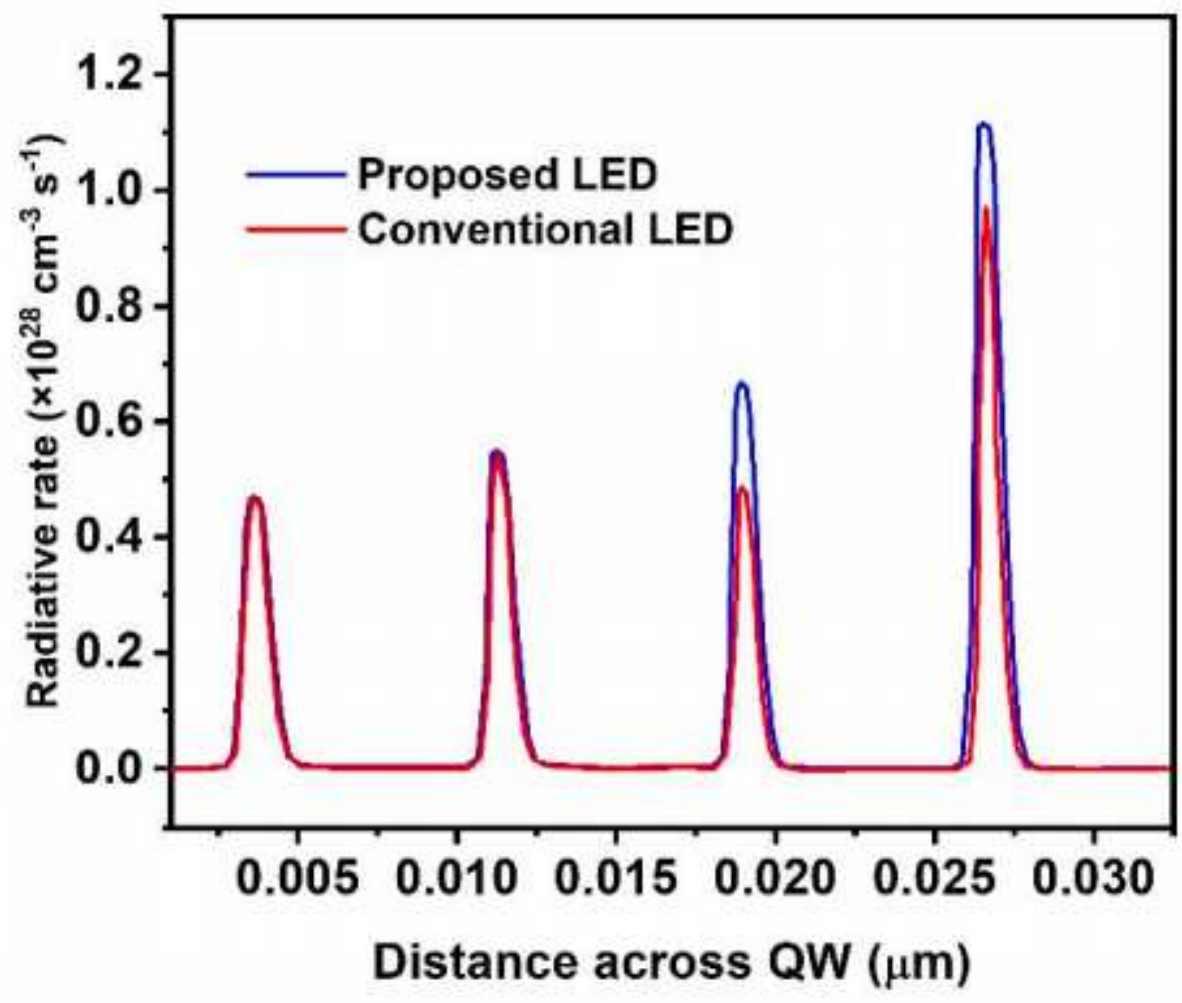

Figure 4

Radiative recombination rate across quantum well for conventional and proposed LED.

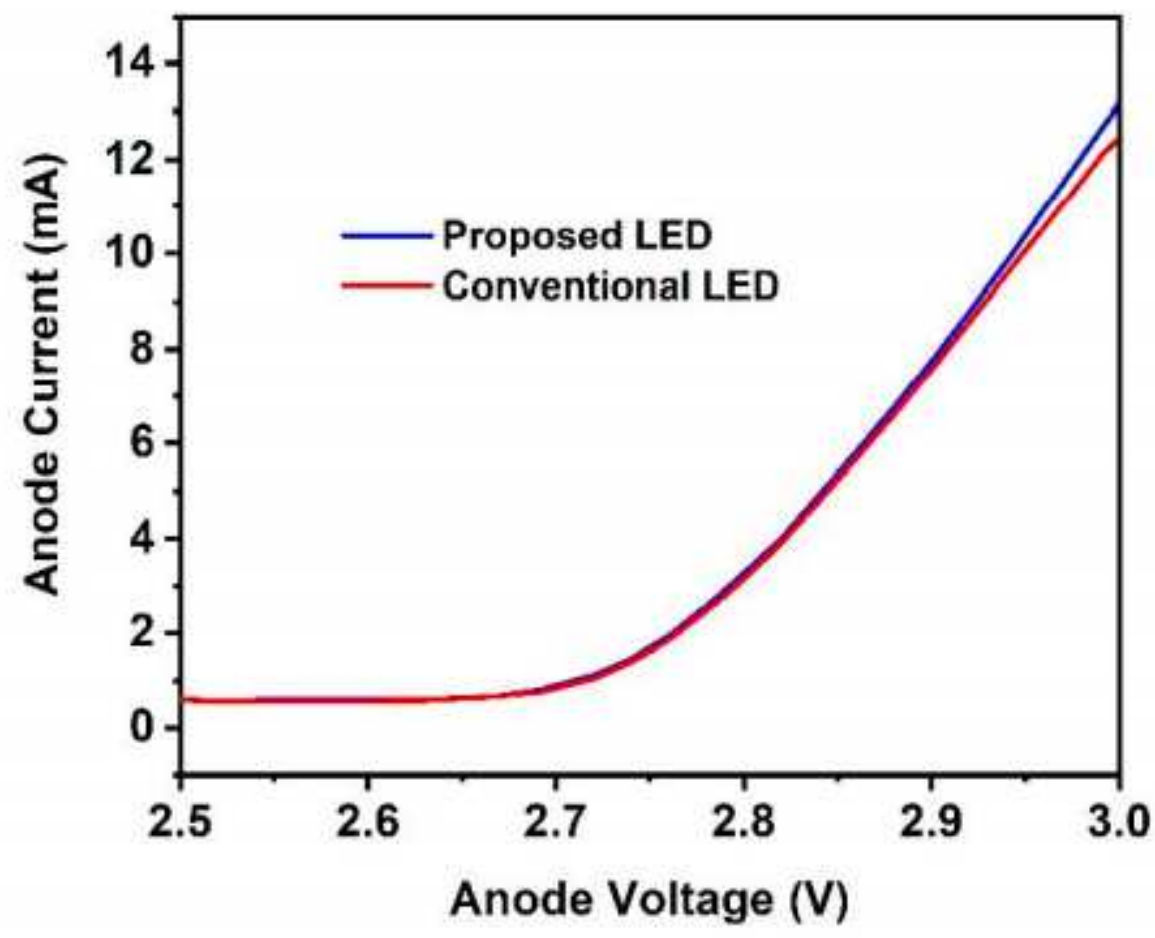

Figure 5 
Anode current at forward bias condition of the LED

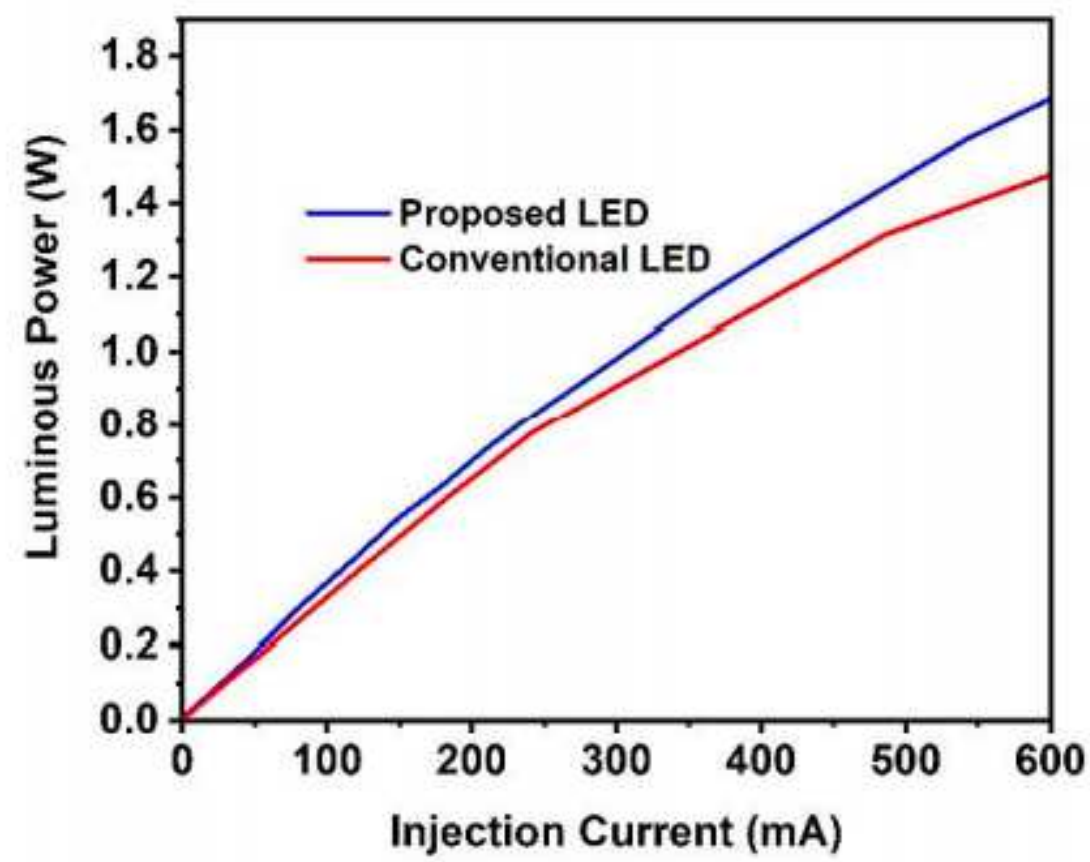

Figure 6

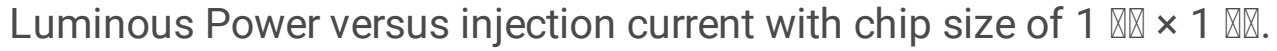

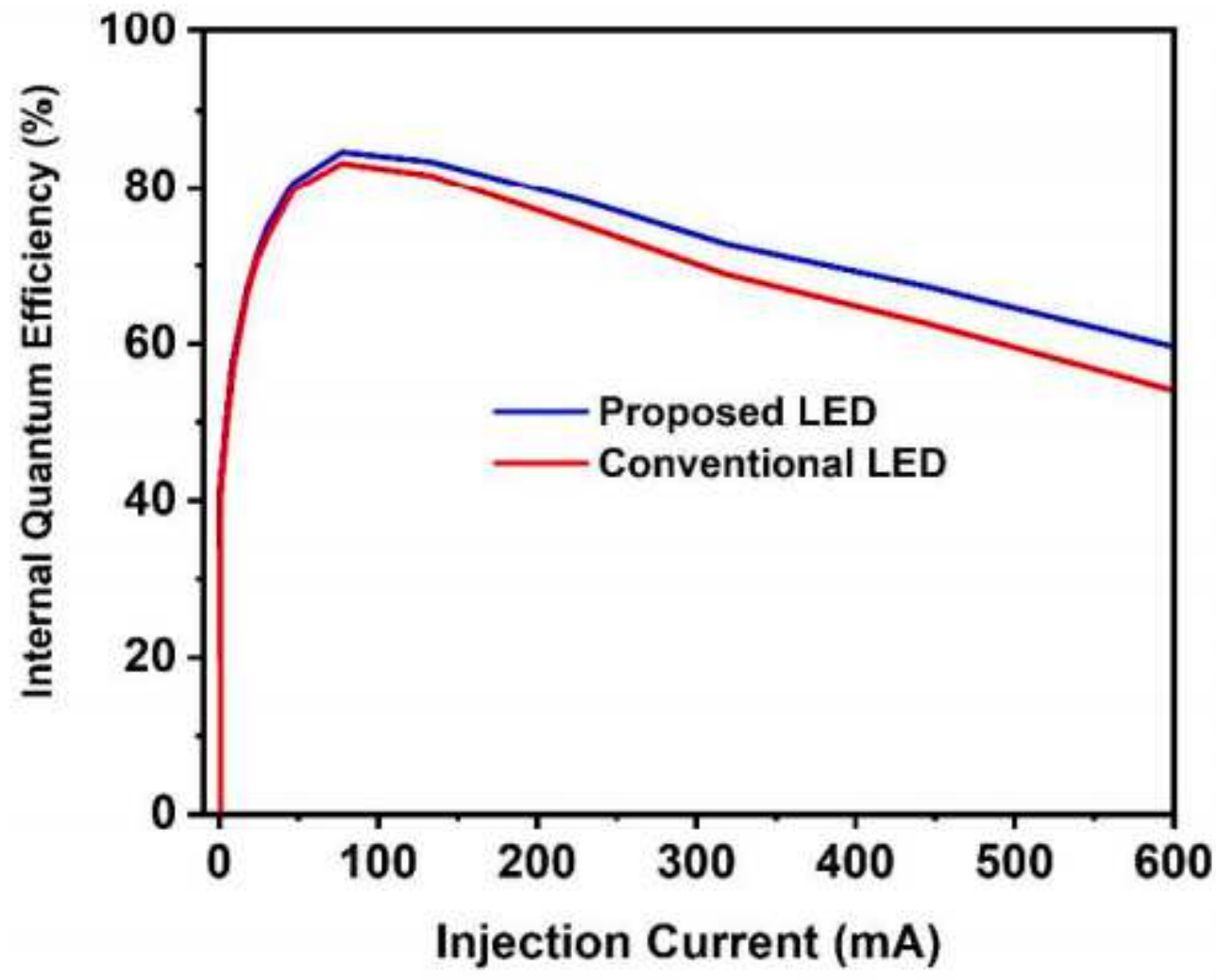

Figure 7 


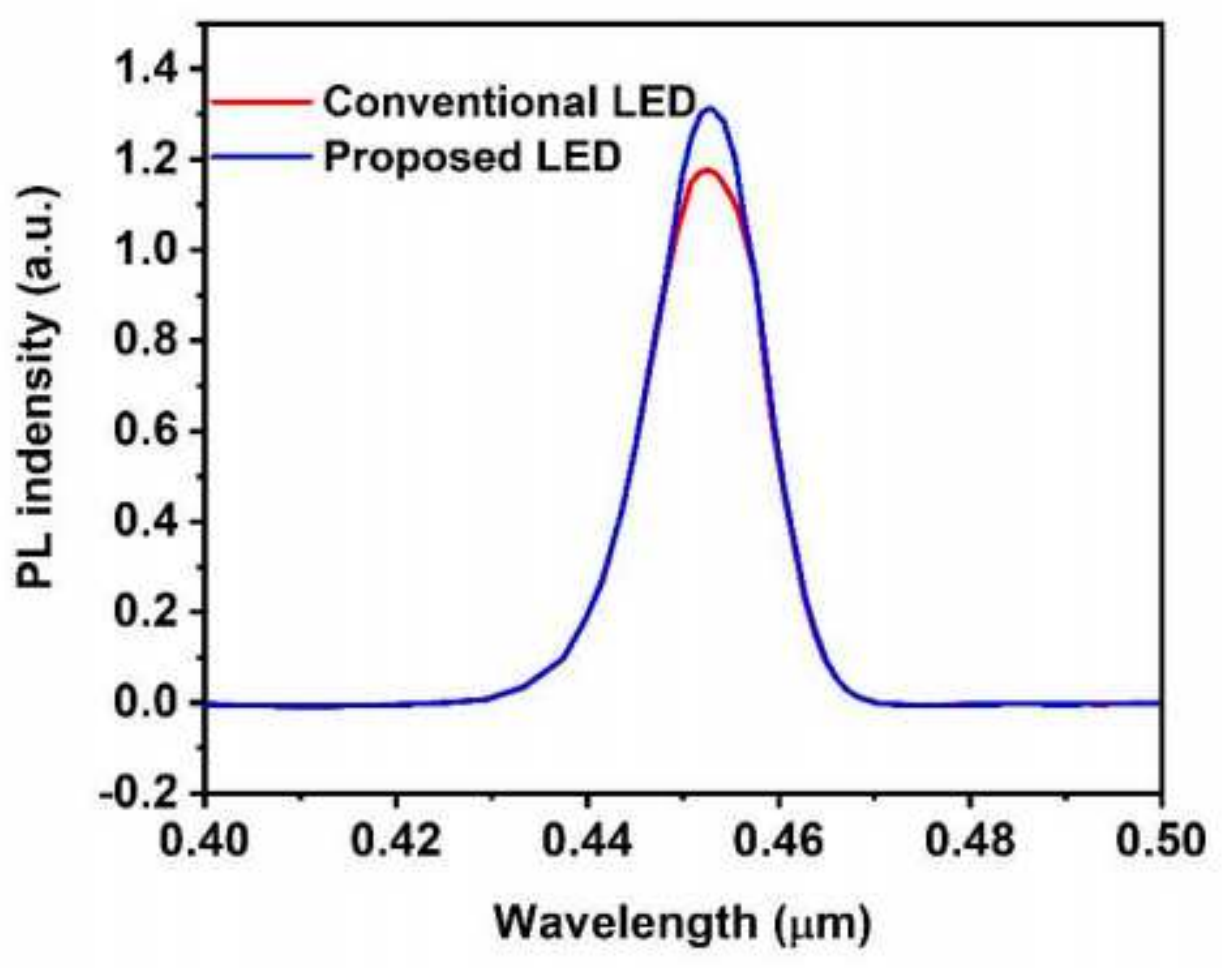

Figure 8

Photo-luminous $(\mathrm{PL})$ intensity versus wave length of the LED. 